\title{
Matryoshka: A Coalesced Delta Sequence Prefetcher
}

\author{
Shizhi Jiang \\ shizhi@nfs.iscas.ac.cn \\ University of Chinese Academy of Sciences \\ Institute of Software, Chinese Academy of Sciences \\ Beijing, China \\ Qiusong Yang \\ qiusong@iscas.ac.cn \\ Institute of Software, Chinese Academy of Sciences \\ Beijing, China
}

\begin{abstract}
To learn complex memory access patterns effectively, many spatial data prefetchers have been proposed that characterize the patterns as fixed-length delta sequences. However, because complex patterns are variable in workloads, it is difficult for fixed-length delta sequences to recognize them with both high competitive coverage and accuracy. That is, longer delta sequences increase accuracy at a lower probability of pattern matching, while shorter delta sequences increase coverage at a higher probability of false predictions. A classical strategy is to introduce the multiple matching mechanism associated with variable-length delta sequences, but sequences have to be redundantly stored in multiple tables.

We observe that shorter delta sequences can coalesce into longer sequences of a fixed length during learning processes. At the same time, the shorter ones can be extracted from the longer ones during matching processes. By leveraging this property, we can improve the storage efficiency of the multiple matching mechanism. In this paper, we propose a novel low-overhead prefetcher, named Matryoshka, that supports the multiple matching mechanism with high efficiency. Instead of maintaining variable-length delta sequences in multiple tables, Matryoshka coalesces variable-length delta sequences into fixed-length delta sequences, which can be maintained with a single pattern table. Concerning the evaluation of a simulated single-core system using memory-intensive workloads of SPEC 2017, Matryoshka outperforms the state-of-the-art prefetcher IPCP by $6.5 \%$ and surpasses SPP+PPF by $2.9 \%$ with $26 \times$ lower storage overhead.
\end{abstract}

ACM Reference Format:

Shizhi Jiang, Yiwei Ci, Qiusong Yang, and Mingshu Li. 2021. Matryoshka: A Coalesced Delta Sequence Prefetcher. In 50th International Conference on Parallel Processing (ICPP '21), August 9-12, 2021, Lemont, IL, USA. ACM, New York, NY, USA, 11 pages. https://doi.org/10.1145/3472456.3473510

Permission to make digital or hard copies of all or part of this work for personal or classroom use is granted without fee provided that copies are not made or distributed for profit or commercial advantage and that copies bear this notice and the full citation on the first page. Copyrights for components of this work owned by others than ACM must be honored. Abstracting with credit is permitted. To copy otherwise, or republish, to post on servers or to redistribute to lists, requires prior specific permission and/or a fee. Request permissions from permissions@acm.org.

ICPP '21, August 9-12, 2021, Lemont, IL, USA

(C) 2021 Association for Computing Machinery.

ACM ISBN 978-1-4503-9068-2/21/08 . .\$15.00

https://doi.org/10.1145/3472456.3473510

\author{
Yiwei Ci \\ yiwei@iscas.ac.cn \\ Institute of Software, Chinese Academy of Sciences \\ Beijing, China
}

Mingshu Li

mingshu@admin.iscas.ac.cn

Institute of Software, Chinese Academy of Sciences

Beijing, China

\section{INTRODUCTION}

The technique of data prefetching plays a vital role in addressing the issue of Memory Wall[34]. Some earlier prefetchers aim to address simple memory access patterns, such as prefetching consecutive addresses after the current miss address[12, 16, 30] or the next few addresses with the same stride[10, 11, 14, 25]. Different from prefetching simple patterns, dealing with complex patterns with varied spatial correlations is one of the main obstacles for modern data prefetchers. In recent years, many spatial data prefetchers (SDPs) $[6-9,13,15,17,18,20,24,29]$ have been proposed, which aim to accurately predict complex patterns with limited on-chip storage. Based on (but not limited to) the past spatial correlations in a region, the SDPs rely on predicting next deltas or offsets relative to the current access.

One of the most efficient methods for SDPs is to capture complex patterns separately using delta sequences[7, 17, 24, 29]. These prefetchers leverage a mechanism named the recursive lookahead mechanism (RLM) to recursively calculate the next delta sequence based on previous sequences to reconstruct a complete pattern. For a delta sequence consisting of $d$ ordered deltas in a region (e.g., $-10,-3,10,-14$ in the page $A$ ), the first $d-1$ deltas are used as the prefix to map the last delta during prefetching. Unlike the other methods[6, 13, 18, 20,31], RLM records the deltas' order in a pattern and allocates the space for storing as required. Meanwhile, because different patterns can share the common sequences, the prefetchers based on RLM usually require a relatively small overhead.

A key issue for prefetchers based on RLM is to configure the best length $d$ for sequence matching. That is, longer delta sequences increase prefetch accuracy at a lower probability of pattern matching, while shorter delta sequences increase prefetch coverage at a higher probability of false predictions. In addition to this, the appropriate length can be uncertain in different workloads. Instead of using the single matching strategy in RLM, Shevgoor et al. proposed VLDP [29] that attempts to predict patterns by matching the current access sequence with multiple delta sequences of different lengths. The variable-length delta sequences are stored straightly in multiple prediction tables, where each table stores sequences of a certain length. VLDP accepts the longest matched sequence to ensure accuracy at the cost of maintaining redundant sequences. By contrast, we will introduce a new multiple matching mechanism with only one table by coalescing the variable-length delta sequences into fixed-length delta sequences. We define sequences 
after coalescing as Coalesced Delta Sequences. The point is to ensure the integrity of short delta sequences in a coalesced delta sequence so that they can be extracted without loss of information. Ultimately, we suggest storing the delta sequences in the primitive form as a return to simplicity.

In this paper, we propose Matryoshka, a prefetcher employing the multiple matching mechanism to capture complex patterns. Instead of maintaining variable-length delta sequences in multiple prediction tables, Matryoshka coalesces them into fixed-length delta sequences, then stores them in one pattern table. Matryoshka demonstrates simultaneous coverage and accuracy improvements with limited storage. The main contributions of this paper are as follows:

- We propose an efficient multiple matching mechanism based on coalesced delta sequences with only one pattern table, which reduces the storage overhead and simplifies the pattern recording process.

- In company with the multiple matching mechanism based on coalesced delta sequences, we devise an adaptive voting strategy to filter out irrelevant prefetch candidates to ensure high prefetch accuracy. Additionally, we propose a novel dynamic indexing strategy to maintain high frequency portions of metadata and implement the architecture at the storage cost of nearly $1.79 \mathrm{~KB}$.

- We use the ChampSim simulator to evaluate Matryoshka in single-core and multi-core systems. The results show that Matryoshka offers a $53.1 \%$ speedup over the non-prefetching baseline in the single-core system, while also outperforms the state-of-the-art prefetcher by $6.5 \%$. In the multi-core system, Matryoshka offers a $32.2 \%$ speedup over the baseline.

The remaining sections are organized as follows. Section 2 describes the background about RLM in detail and introduces several state-of-the-art RLM-based prefetchers. Section 3 discusses the motivations. Section 4 describes the innovative mechanisms of Matryoshka. Further, Section 5 describes the hardware implementation of Matryoshka in detail. An exhaustive performance evaluation is presented in Section 6. Finally, we conclude the paper in Section 7.

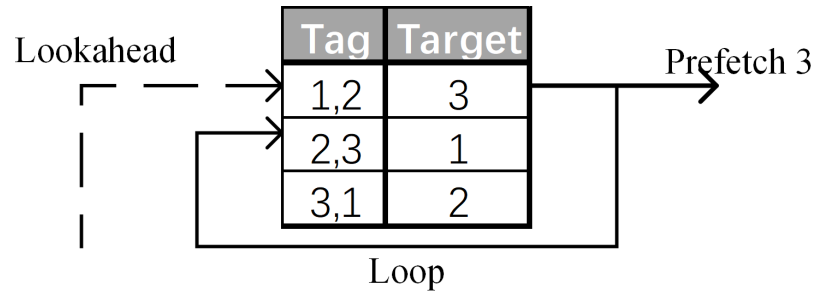

Figure 1: Recursive lookahead mechanism.

\section{BACKGROUND}

Generally, the lengths of complex patterns in workloads are varied, which creates difficulties for describing complex patterns. The shortest complex pattern consists of only two deltas, e.g., $<1,-2>$. If an address $A$ triggers this pattern, then $A+1$ and $A-1$ will be sequentially prefetched. On the other hand, a long complex pattern can consist of dozens of unique deltas. To avoid describing a whole pattern at a time, RLM-based prefetchers use a snapshot window in fixed-length to separately record a pattern as many delta sequences. During the learning phase, the snapshot window keeps sliding to the next delta and records every sequence in the window. For example, with the snapshot window of size two, the trace $1,2,3 \ldots$ will be captured as $(1,2),(2,3)$ and so on. During prefetching, for replaying a pattern of $n$ deltas, $n$ sequences are needed no matter the length of sequences. Fig. 1 shows the procedure of restoring a pattern in RLM. In the figure, the delta sequences $(1,2,3),(2,3,1)$ and $(3,1,2)$ are required to maintain the pattern $\langle 1,2,3\rangle$ in RLM. A current access sequence $(1,2)$ can match the prefix $(1,2)$ of $(1,2,3)$ and continue to the next matching process after being updated (e.g., $(1,2) \rightarrow(2,3))$. It is necessary to keep these three sequences for the pattern $<1,2,3>$, which form a loop, to drive the recursive lookahead process at any starting point.

In recent years, many prefetchers based on RLM have been proposed. The Variable Length Delta Prefetcher (VLDP)[29] intuitively implements the multiple matching mechanism using three fullyassociative prediction tables, and prefers to make predictions based on the longest matched sequence for high accuracy. We argue that its structure of multiple tables maintains redundant sequences, and its accuracy decreases due to ignoring the short matches. Matryoshka presented in this paper will address these issues. The Signature Path Prefetcher (SPP)[17] computes compressed signatures to index predicted deltas, and controls the prefetch degree according to the probability calculated through the prefetch path. SPP uses sequences consisting of five deltas to capture patterns accurately in theory. However, the accuracy decreases because the 4-delta prefix ( $4 \times 7$ bits) of a sequence is compressed as a coarsegrained signature (12 bits). The Perceptron-based Prefetch Filtering (PPF)[7] proposes an intelligent filter to enhance SPP. PPF obtains high performance by filtering the useless prefetches issued by an aggressive SPP, but Matryoshka achieves higher accuracy with a much lighter strategy. Pangloss[24] is another state-of-the-art, heavy prefetcher based on the Markov chain theory. Unlike SPP using a compressed signature to index following deltas, Pangloss uses only one fine-grained delta (10 bits) to index them. Pangloss uses the delta to index a big table (1024 sets) to avoid hash conflicts, which constructs a bijection between deltas and sets. Although the storage overhead of Pangloss is very large, it can have trouble tracking long complex patterns.

\section{MOTIVATION}

In this section, we first discuss the obstacles in describing complex patterns with delta sequences. We believe the multiple matching mechanism can overcome these issues. To efficiently support this mechanism, we explore the efficiency of different forms for storing delta sequences. Finally, we analyze the distribution of deltas in workloads aiming at a further reduction of the storage.

\subsection{Capturing Complex Patterns}

Usually, many different memory access patterns and noises (nonrepetitive accesses) are mixed during program execution. In addition, memory access instructions are not always executed in program order in modern out-of-order CPUs. The patterns of a program can be elusive because of these factors, which creates great 


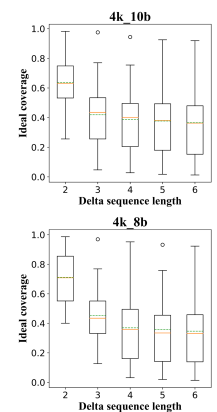

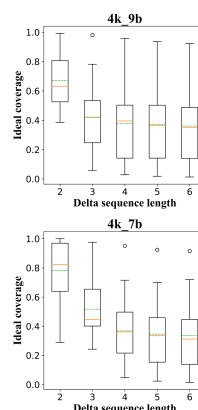

(a) (b)

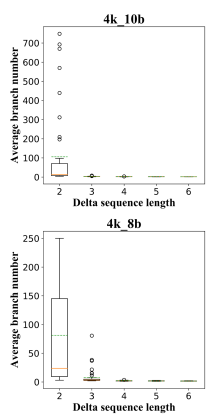

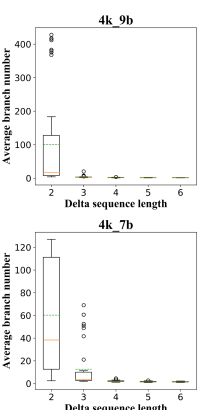

Figure 2: Delta sequences of variable-lengths (from 2 deltas to 6 deltas) are separately collected in $4 \mathrm{~KB}$ pages in 45 traces, and the delta widths vary from 10 bits to 7 bits. The green dotted lines indicate the averages of the distributions and the yellow lines indicate the medians. (a)The results show that the longer the sequence, the lower the chance of recurrence. Therefore, the ideal coverage is shrinking while increasing the delta sequence length. (b)Average branch numbers are very low when the length of delta sequences is 3 and the delta width is bigger than 9 bits. When the delta width is 8 bits or 7 bits, one more delta is needed to limit the mutation in a small range.

difficulties for accurate pattern learning. RLM-based prefetchers usually select the best length of delta sequences in statistics of workloads to achieve high performance. To find the best length, we measure the theoretical performance of the delta sequences of different lengths and different delta widths ${ }^{1}$ for capturing patterns through a trivial experiment. For convenience, the following two metrics are defined to measure the theoretical performance:

Ideal Coverage is the proportion of fixed-length delta sequences appearing at least twice in a workload. Intuitively, a sequence which appears only once is not worth prefetching ${ }^{2}$.

An Average Branch Number is the average number of branches of fixed-length delta sequences which appear at least twice in a workload. We say a delta sequence has a branch if its longest prefix (not including itself) is the exact prefix of some other sequences. With the increase of the average branch number, it can be difficult to determine the exact branch, which results in increasing mispredictions.

We analyze the two metrics of 45 traces captured from the SPEC 2017 workloads[3]. Fig. 2(a) shows the distributions of the ideal coverage in traces for delta sequences of different lengths, and Fig. 2(b) presents the distributions of the average branch numbers in traces for delta sequences of different lengths. According to the quantitative analysis, we can reduce the branches of accessing sequences significantly by increasing the length of delta sequences to four deltas. For the shorter delta sequences, there can be ambiguity in determining possible accessing patterns. In fact, it is more challenging to find a suitable length providing high ideal coverage in all

\footnotetext{
${ }^{1}$ The delta width is the number of bits for a delta, which controls its range (grain). For example, 7-bit deltas in $4 \mathrm{~KB}$ pages can describe the spatial correlations between cache blocks.

${ }^{2}$ Please note that it does not indicate that compulsory misses are not worth prefetching. Because sequences are a form of memory access patterns, it should not be prefetched if a pattern does not repeat.
}

workloads. Fig. 2(a) shows that the average ideal coverage among the traces is great with 2-delta sequences. When using four deltas, the average ideal coverage drops by about $20 \%$ in any delta width. Moreover, the distributions of the ideal coverage in these traces are scattered no matter the length of sequences. Therefore, we believe it is a better choice for prefetchers to capture complex patterns using variable-length delta sequences.

Ozturk et al.[21] have also observed the benefits of exploiting multiple delta-pattern history lengths. Further, we analyze the effect of delta widths and find that increasing the delta width instead of lengthening delta sequences can achieve higher accuracy with lower consumption. For example, the average branch number for sequences of three 10-bit deltas (30 bits) is similar to sequences of four 8-bit deltas (32 bits), or five 7-bit deltas (35 bits). We will discuss the choices by quantifying the improvement in Section 6.5.

\subsection{Efficiency of Delta Sequences in Different Forms}

One of the advantages of delta sequences is that they exploit the spatial and temporal correlations simultaneously. It is more accurate to capture complex patterns using delta sequences compared with other methods (e.g., footprint [6, 31]), but more costly when representing long patterns. An intuitive idea of reducing storage is to compress the metadata[7, 17, 26-28]. By sacrificing accuracy, the patterns can be stored at lower overhead with compressed delta sequences. However, we find that it is possible to reduce the overhead without decreasing accuracy. Based on coalesced delta sequences, we can build a storage-efficient multiple matching mechanism with lossless history. The prefixes of different lengths from a coalesced sequence can be extracted as sub-sequences for multiple matching during prefetching. The detailed design will be described in Section 4 . As a result, every entry of a table contains several sequences in theory, which is more efficient.

For the sake of intuition, we attempt to prove the high efficiency of coalesced sequences over other forms by algebra. We first define the information density as the average number of delta sequences per bit and omit the last delta (predicting target) of a delta sequence in the following analysis for simplicity. Let the positive value $\alpha$ be the compression ratio, $n$ be the number of deltas in a sequence, and $b$ be the bits of the delta. In the single matching mechanism, the information density is always $1 /(\alpha n b)$, where $\alpha \leq 1$. In the conventional multiple matching mechanism, there are $m$ sequences from the 1-delta sequence to the $n_{m}$-delta sequence for matching. The bits for a (compressed) sequence with $n_{i}$ deltas are $\alpha b n_{i}$, so the overall information density for $m$ sequences is $m /\left(\alpha b \sum_{i=1}^{m} n_{i}\right)=2 m /\left(\alpha b n_{m}\left(n_{m}+1\right)\right)=2 /(\alpha b(m+1))$, where $n_{i}$ represents the number of deltas used for the $i$ th sequence, $m$ is the number of sequences for multiple matching, $n_{m}=m$ and $\alpha \leq 1$. In our design, only the prefixes of a coalesced delta sequence are used as tags for matching. That is, $m$ deltas are required to store $m$ variable-length sequences. Only one coalesced sequence is required to represent these sequences. Finally, the information density is $m /(\alpha m b)=1 /(\alpha b)=1 / b$, where $\alpha=1$. Coalesced sequences are not compressed to achieve both the best information density and the finest granularity. According to the algebraic formulas above, VLDP needs to pay $(m-1) / 2$ times more of the storage overhead 


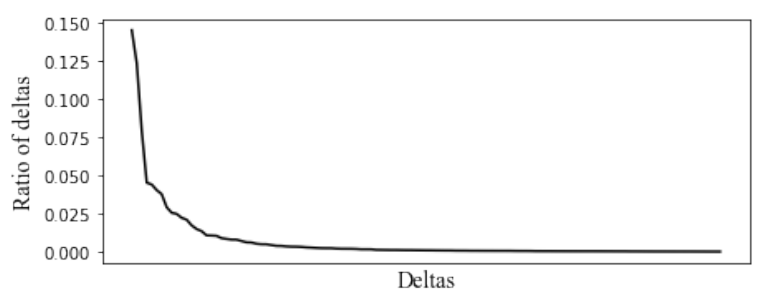

Figure 3: Distribution of the total 10-bit deltas (-511 511) in 45 traces. The data is ordered by ratios of deltas, where ratios below 0.0005 are omitted.

at the same granularity as Matryoshka, where $m>1$. For example, VLDP requires 1-delta, 2-delta, and 3-delta prefixes to support the multiple matching mechanism when $m=3$. By contrast, Matryoshka requires only 3 -delta prefixes maintained. VLDP pays $1 \times$ more storage in theory.

\subsection{Storing Hot Patterns}

The conventional SDPs require thousands of entries for high performance to adapt to the variance of complex patterns. There are several reasons for such storage consumption. First, unlike constant stride patterns, variable strides are required to seize a complex pattern. Not only can different combinations of strides generate different patterns, but different permutations of strides also increase the number of patterns. In addition, it is significant to maintain the auxiliary information such as PCs, page addresses, and access delays that correspond to a pattern, because the information is usually used as signatures to trigger the pattern or control its prefetch timing. Finally, the distribution of patterns is often uneven. Assuming that patterns are stored in set-associative tables, the entries in one set can be inadequate when a large number of patterns with different signatures compete for the same set due to hash conflicts. But using more ways instead to adapt to the uneven distribution produces much more overhead.

The SDPs usually index pattern tables statically by the auxiliary information or deltas, or combinations of them. To reduce the probability of hash conflicts during indexing, the number of table sets must be relatively large. Many powerful prefetchers require more than $16 \mathrm{~K}$ entries to store patterns, resulting in a larger storage requirement than a typical $\mathrm{L} 1$ data cache. Inspired by $\mathrm{Wu}$ et al.'s work[33] which contends that only a small portion of the metadata is frequently reusable, we examine the distribution of high-frequency deltas (10 bits) in the 45 traces captured from SPEC 2017. Fig. 3 displays the impressive results. As shown, most of the deltas rarely appear during the program execution. The top 20 highfrequency deltas account for $74.0 \%$ of the total occurrences. It is possible to reduce the storage by only maintaining a small portion of deltas and their corresponding patterns on a chip. By changing the conventional indexing strategy into the dynamic indexing strategy, we successfully reduce the storage further, which will be described in detail in the next section.

\section{DESIGN}

In this section, we describe the design of Matryoshka, a prefetcher efficiently supporting the multiple matching mechanism based on

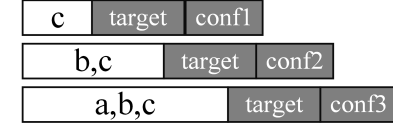

Store 3 sequences separately

$$
\text { set1 }
$$

\begin{tabular}{|c|c|c|c|c|}
\hline $\mathrm{d}$ & b & c & target & 1 \\
\hline a & b & c & target & 4 \\
\hline
\end{tabular}

set0

\begin{tabular}{|c|c|c|c|c|c|c|c|c|c|}
\hline c & $\mathrm{b}$ & $\mathrm{a}$ & target & 4 & c & $\mathrm{b}$ & $\mathrm{d}$ & target & 1 \\
\hline
\end{tabular}

(2)

Figure 4: Differences between forms of delta sequences. Figure (1) compares forms of separate-stored delta sequences and coalesced delta sequences. Figure(2) shows the distribution of coalesced delta sequences in a table after reversing.

coalesced delta sequences, which aims at improving both coverage and accuracy.

\subsection{Reversed Coalesced Delta Sequences}

To improve the efficiency of the multiple matching mechanism, we attempt to merge variable-length delta sequences into a single fixed-length delta sequence. Fig. 4 shows the differences between forms of delta sequences. For the classical method, as the left figure of Fig. 4(1) shown, each entry represents one delta sequence in the compressed form for storage reduction. Coalesced delta sequences explicitly store deltas instead, as shown in the right of Fig. 4(1). Any sub-sequence can be extracted effectively using masks for multiple matching.

During the training phase, a prefetcher usually records the number of times a sequence repeated to form a (sequence, confidence) pair. The confidence is used to calculate the prediction probability of the target delta associated with the matched sequence at prefetching. If we store patterns with sequences of variable lengths, then every sequence needs a distinct confidence counter.

However, it is unnecessary to store confidences for every subsequence in coalesced delta sequences. Only one confidence is enough for a coalesced sequence. As shown in Fig. 4(1), the short sequences $(c)$ and $(b, c)$ are "coalesced" into the sequence $(a, b, c)$, and their confidences conf1 and conf2 are discarded if they are the same as conf3. On the one hand, if the short sequence always recurrences in the same sequence, its confidence is simply the same as the sequence containing it. On the other hand, if the short sequence recurrences more frequently than the sequence containing it, like reappearing with various deltas, there must be multiple entries containing the short sequence that each records a portion of its confidence. Therefore, their confidences can be calculated by accumulating the confidences of the entries containing them For example, $(a, b, c)$ with a confidence of four and $(d, b, c)$ with a confidence of one can tell that $(b, c)$ repeated four and one times along with different deltas, while the confidence of $(b, c)$ is five as shown in Fig. 4(2). 


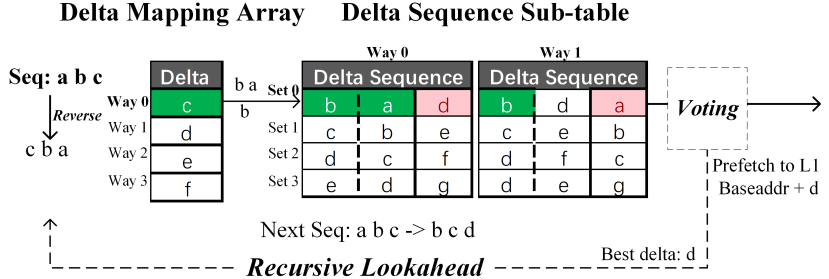

Figure 5: A simplified example using the dynamic indexing strategy. Supposing all confidences are one, and they are omitted.

Ultimately, the coalescing process for sequences and their confidences can be accomplished passively without any specific operation. As long as maintaining a sequence and its confidence, its sub-sequences and their confidences are also recorded. This design eases the logic of counting during training and reduces storage overhead.

To further reduce hardware costs, the coalesced delta sequences will be reversed before being stored. Without reversing, the sequences with the same recent deltas could be scattered in different sets in a table. In this case, the multiple matching mechanism cannot work in parallel without multiple read ports for one request, which brings much more access pressures under the recursive lookahead circumstance. As illustrated in Fig. 4(2), the bottom sub-figure shows the metadata layout of coalesced sequences after reversing. By indexing with the same delta $c$, the sequences $(c, b, a)$ and $(c, b, d)$, transformed from the sequences $(a, b, c)$ and $(d, b, c)$, can be stored in the same set, which avoids multiple sets traversing for one request during prefetching. Please note the predicting targets of sequences are not involved in the reversing operation.

\subsection{Dynamic Indexing Strategy}

Because most of the deltas in workloads rarely appear according to the analysis in Section 3.3, we contend to reduce the storage further by only storing sequences with high-frequency deltas. To achieve this goal, we have to change the conventional indexing strategy. The conventional strategy is to statically bind a record to a fixed index based on its signature (e.g., a part of delta sequences). Because of hash conflicts, records with different signatures can compete fiercely for occupying a few sets in the table. Instead of using the static strategy, we attempt to dynamically map a record to an index at running time by explicitly storing its signature, extended with a confidence field. The (signature, confidence) pairs are stored in an isolated fully-associative structure, in which one with the lowest confidence will be evicted during the learning phase. Therefore, the mapping relation is dynamically built between the pair and its entry index to ensure the reservation of high-frequency signatures. The dynamic indexing strategy can benefit any spatial prefetcher, while Matryoshka achieves a lower cost with it due to its high information density.

Fig. 5 illustrates the process of the multiple matching mechanism with 2 and 3-delta prefixes of stored 4-delta sequences. For a 4-delta sequence, the order of the first three deltas is reversed, while the fourth delta (target) remains the same. To reserve the high-frequency deltas, Matryoshka uses the first delta of a reversed sequence as the signature to index the following sequence based on the dynamic indexing strategy. Matryoshka constructs the pattern table with two gadgets to support the strategy. The Delta Mapping Array (DMA) keeps the mapping relations between deltas and indexes of sets, and the Delta Sequence Sub-table (DSS) keeps the following sequences in associated sets. In other words, the matching DMA way number is used as a set number to DSS. During the prefetching phase, the first delta of the current accessing sequence is used to search the delta-index relations in DMA, then the rest part of the sequence is compared with the prefixes of reversed coalesced delta sequences stored in associated sets of DSS.

For instance, the current accessing sequence $(a, b, c)$ is used to match as illustrated in Fig. 5. First, the sequence is reversed to $(c, b, a)$, then the first delta $c$ hits Way 0 in DMA. Second, $(b, a)$ and (b) are used to match the 2-delta and 1-delta prefixes of sequences in Set 0 of DSS. They hit entries Way 0 and Way 1 of Set 0 in DSS as the green cells shown. After voting, the candidate delta $d$ illustrated in the pink cell at Way 0 of Set 0 is selected after matching. Descriptions for the voting strategy will be given in the next sub-section.

\subsection{Adaptive Voting Strategy}

Different from prefetchers storing tag-target pairs with unique tags, Matryoshka maintains unique coalesced sequences including target deltas. Therefore, there could be multiple sequences with the same tag but different targets, or with the same target but different tags. This design could help provide the voting phase with as many voters as possible and increase prefetching opportunities.

Our key idea for accurate voting is to both consider the confidences of the short and long sequence matches, but with different weights to adaptively fit the lengths of matched sequences. Because we can restore the confidence of a short sequence by accumulating the confidences of entries containing it, any delta can be selected if its score, which is the sum of products of the confidences of matched sequences and the corresponding weights, satisfies the prefetch criterion. In our design, the candidate delta associated with the matched sequence is selected if the ratio of its score to the total score of all candidates exceeds the prefetch threshold.

Let Score $_{d}$ be the score of a candidate delta $d$, and Score total be the total score of candidate deltas. They can be calculated as follows:

$$
\begin{aligned}
\text { Score }_{d} & =\sum_{i \in L} W_{i} \sum_{j \in M_{i}} \operatorname{Conf}_{j} \\
\text { Score }_{\text {total }} & =\sum_{d \in D} \text { Score }_{d}
\end{aligned}
$$

where $W_{i}$ is the weight for each matched prefix of length $i ; L$ is the set of the lengths; $M_{i}$ indicates the entries, containing the matched prefixes of length $i$, that generate $d$; $\operatorname{Con} f_{j}$ is the confidence of the corresponding entry $j$; $D$ is the set of candidate deltas. Here is the judgment formula:

$$
\frac{\text { Score }_{d}}{\text { Score }_{\text {total }}}>T_{p}
$$

where $T_{p}$ is the prefetch threshold

For the sake of intuition, we give some examples to illustrate the calculation process of voting. For example, suppose that the current delta sequence $(c, b, a)$ is ready to match the coalesced sequence $(c, b, a)$ and $(c, b, d)$ that share the same Target in Fig. 4(2). Because 
they can be matched by the current sequence and its prefix $(c, b)$ respectively, the score of Target is $4 \times W_{3}+1 \times W_{2}$, where 4 is the confidence of $(c, b, a)$ and 1 is the confidence of $(c, b, d)$. After summing the confidences of all the matched sequences, we can calculate the ratio for Target. If the ratio exceeds $T_{p}$, it is selected as a prefetch candidate. Otherwise, if the matched coalesced sequences have different target deltas, their scores should be calculated separately. For the example in Fig. 5, the score of target $d$ is $1 \times W_{3}$ and the score of target $a$ is $1 \times W_{2}$, where their confidences are both 1 . Assuming $W_{3} /\left(W_{3}+W_{2}\right)>T_{p}$, then the address $($ Baseaddr $+d)$ is prefetched. The computation process for each target delta can be achieved in parallel.

\subsection{Discussion}

4.4.1 Is it necessary to reverse sequences? By reversing, Matryoshka can gather coalesced sequences with the same recent delta into the same set in a table, which eliminates the requirement of multi-set traversing and leverages the temporal locality of memory accesses. Some may ask to support multiple matching using not the suffix but the prefix of a sequence in normal order as the indexing key, e.g., $a$ for $(a, b, c)$, so that they also hit the same set. But the timelines of these prefixes are not aligned, which impacts the prefetch timeliness. To improve the timeliness of prefetches, Matryoshka prefers to match recent deltas nearing targets. According to our experiments, the late prefetches using reversed sequences are less than using sequences in the original order.

4.4.2 Access Latency. The access latency of Matryoshka can be enlarged due to the dynamic indexing strategy. We believe the impact can be alleviated by implementing Matryoshka through a pipeline structure, by which the access time can be amortized. Due to the limitation of the simulator, we will implement the precise pipeline structure in our future research ${ }^{3}$.

4.4.3 Redundancy. We notice that although Matryoshka reduces the redundancy of metadata by coalescing, it is not eliminated. The redundancy is introduced intrinsically by RLM for restoring patterns, e.g., duplicated parts of sequences for concatenating patterns in Fig. 1. The longer the sequence, the more the redundancy of this type.

\section{IMPLEMENTATION}

In this section, we present the implementation of Matryoshka in detail. First, we introduce the overview of the architecture. Then, we elaborate on the learning phase and prefetching phase of Matryoshka. Finally, we enumerate some of the important optimizations.

\subsection{Architecture Overview}

Matryoshka aims to predict patterns within $4 \mathrm{~KB}$ physical pages (12 bits offset). The patterns are stored as the coalesced delta sequences consisting of four 10-bit deltas $(-511 \sim 511)$ in which the high seven bits of deltas are required for prefetching cache blocks (64B) to balance the coverage and accuracy. Based on this structure,

\footnotetext{
${ }^{3}$ Many state-of-the-art prefetchers did not claim their access time (e.g., SPP+PPF Pangloss), so all prefetchers simulated in our experiments are under a loose latency limitation for comparing their ideal performance.
}

Table 1: Detailed Storage Overhead of Matryoshka

\begin{tabular}{|c|c|c|c|}
\hline Structure & Entry & Field & Storage \\
\hline History Table & $128 \times 1$ & $\begin{array}{c}\text { PC tag (12 bits) } \\
\text { Page tag ( } 8 \text { bits) } \\
\text { Last offset ( } 9 \text { bits) } \\
\text { Last delta sequence } \\
\text { (30 bits) } \\
\text { Valid (1 bit) }\end{array}$ & 7680 bits \\
\hline $\begin{array}{c}\text { Delta Mapping } \\
\text { Array }\end{array}$ & $1 \times 16$ & $\begin{array}{c}\text { Delta (10 bits) } \\
\text { Confidence ( } 6 \text { bits) } \\
\text { Valid (1 bit) }\end{array}$ & 272 bits \\
\hline $\begin{array}{l}\text { Delta Sequence } \\
\text { Sub-table }\end{array}$ & $16 \times 8$ & $\begin{array}{c}\text { Delta sequence (30 bits) } \\
\text { Confidence ( } 9 \text { bits) } \\
\text { Valid ( } 1 \text { bit })\end{array}$ & 5120 bits \\
\hline Candidate Array & $128 \times 1$ & Score (10 bits) & 1280 bits \\
\hline $\begin{array}{c}\text { Candidate Offset } \\
\text { Array }\end{array}$ & $32 \times 1$ & Score (10 bits) & 320 bits \\
\hline \multicolumn{4}{|c|}{ Total $: 14,672$ bits $\approx 1.79 \mathrm{~KB}$} \\
\hline
\end{tabular}

Matryoshka supports the multiple matching mechanism with delta sequences of two lengths, the 3 and 4-delta sequences (including targets). Matryoshka learns patterns from L1 load accesses and prefetches data into L1 according to the formula 2 with the threshold $T_{l 1}=0.5$. In other words, Matryoshka only allows one prefetch at most for each turn in the process of RLM.

There are three major components in Matryoshka: the History Table (HT), the Delta Mapping Array (DMA), and the Delta Sequence Sub-table (DSS).

- HT, storing the history information captured from L1 load accesses, is a 128-entry direct-mapped table.

- DMA, storing the mapping relationships of deltas and sets, is a 16-entry fully-associative array.

- DSS is a $16 \times 8$-entry set-associative sub-table maintaining a variety of delta sequences and their confidences.

DMA and DSS, separated to enable the dynamic indexing strategy, constitute the Pattern Table (PT). In addition, the Candidate Array (CA, 128-entry) and the Candidate Offset Array (COA, 32-entry) are used to accumulate scores of target deltas for generating prefetch candidates and driving the RLM process. The detailed fields for each component are illustrated in Table 1.

\subsection{Learning Patterns with Coalesced Delta Sequences}

Matryoshka is trained with only L1 loads. Fig. 6 shows a detailed case for the training phase. As illustrated, the process is divided into three steps. (1)HT collects the information of loads localized by PCs and assembles 4-delta sequences. The reversing stage is explicit for easier understanding. In fact, the Last Delta Sequence can be stored in reversed order without a specific reversing operation. The page tag is used to check whether crossing pages happens; if it does, the delta will be revised. As a result, the reversed sequence $(24,22,20)$ is obtained from the records in HT and the target delta 26 is calculated using the current L1 load access and last offset. (2) The first delta 24 is the signature, which hits Way 2 of DMA. Then, the confidence of Way 2 increases by 1 , and the corresponding Set 2 


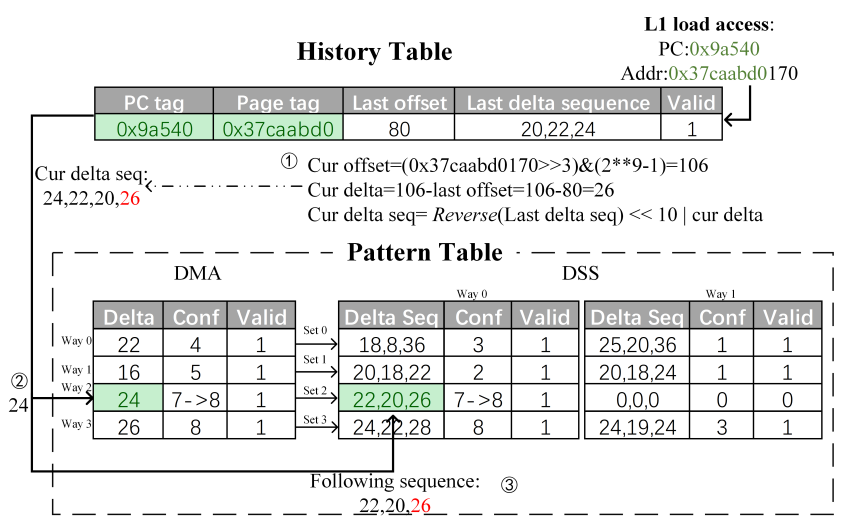

Figure 6: Training phase of Matryoshka

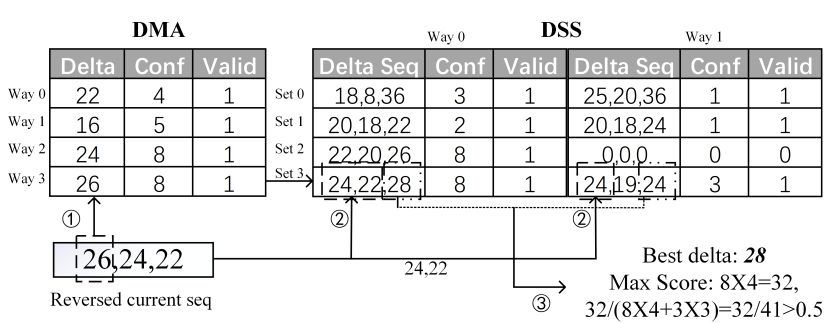

Figure 7: Prefetching phase of Matryoshka.

of DSS will be updated. When the confidence reaches the max, all the other confidences in DMA have to be halved for concentrating on recent sequences, which is slightly different from proportional counters[20]. If no entry hits, the entry with the lowest confidence is replaced by the new delta, and the records in DSS related to the evicted entry are reset correspondingly. (3) Way 0 of Set 2 in DSS is hit by the following sequence $(22,20,26)$, where the confidence of the matched entry increases by 1 . The updating policy for every set in DSS is the same as that of DMA. By replacing the records on the basis of their confidences, only the high frequency records are reserved in PT.

\subsection{Efficient Multiple Matching-based Prefetching}

Matryoshka attempts to strictly trigger prefetching every time a load instruction arrives. The recent accesses are used to match a certain pattern for yielding prefetch requests. Fig. 7 depicts an elaborate example for the process of matching records using the reversed current access delta sequence. The prefetching phase is divided into three steps for each matching process of RLM. (1) First, the reversed current delta sequence $(26,24,22)$ is assembled with the PC-indexed records in HT and the current access. The newest delta 26 is used as the signature to locate the entry in DMA. (2) Delta 26 hits in Way 3. Then, $(24,22)$ and (24) are used to match the corresponding prefixes of the records in Set 3 of DSS. As shown in the figure, the delta 28 and 24 are selected at Way 0 and Way 1 of Set 3. (3) Then, Matryoshka applies the adaptive voting strategy using $\mathrm{CA}$ and $\mathrm{COA}$ to select the final deltas from candidate deltas to prefetch, as described in Section 4.3. We omit the description of the

${ }^{4}$ We do not draw all the sets and ways for simplicity.
Table 2: Simulated System Configuration

\begin{tabular}{|c|c|}
\hline Core & $\begin{array}{l}\text { One to four cores, 4GHz, 4-wide, 352-entry } \\
\text { ROB, 128-entry LQ, 72-entry SQ, } 4 \mathrm{~KB} \text { Page }\end{array}$ \\
\hline TLBs & $\begin{array}{l}\text { 64-entry ITLB, 64-entry DTLB, 1536-entry } \\
\text { L2DTLB }\end{array}$ \\
\hline L1I & $\begin{array}{l}\text { 32KB, 8-way, 32-entry PQ, 8-entry MSHR, } \\
4 \text { cycles }\end{array}$ \\
\hline L1D & $\begin{array}{l}48 \mathrm{~KB}, 12 \text {-way, 8-entry PQ, 16-entry MSHR, } \\
5 \text { cycles }\end{array}$ \\
\hline L2 & $\begin{array}{l}512 \mathrm{~KB}, 8 \text {-way, 16-entry PQ, 32-entry MSHR, } \\
10 \text { cycles }\end{array}$ \\
\hline LLC & $\begin{array}{l}2 \mathrm{MB} \text { to } 8 \mathrm{MB}, 16 \text {-way, } 32 \text { to } 128 \text {-entry PQ, } \\
64 \text { to } 256 \text {-entry MSHR, } 20 \text { cycles }\end{array}$ \\
\hline DRAM & $\begin{array}{l}\text { 4GB } 1 \text { channel (1-core), 8GB } 2 \\
\text { channels (4-core), } 3200 \mathrm{MT} / \mathrm{sec}\end{array}$ \\
\hline
\end{tabular}

implementation of the voting strategy due to space reasons. In our configuration, the voting weights are $W_{2}=3$ and $W_{3}=4$ for the matching lengths of 2 and 3 deltas respectively in the formula 2. If the score's ratio of the selected delta to the total score is greater than $T_{l 1}$, a load instruction is issued to prefetch data into the $\mathrm{L} 1$ cache; otherwise, no prefetch will be issued. According to the computation in Fig. 7(3), the score of delta 28 is 32 , and the total score of all candidate deltas is 41 . To the end, the delta 28 is the best delta with the highest score and its ratio $32 / 41$ is also greater than $T_{l 1}=0.5$. The address (Baseaddr +28 ) is generated to prefetch data into L1.

Finally, for the next matching process, the best delta is appended to the current sequence, and the oldest delta of the sequence is removed. If the ratio of the best delta's score to the total score of all candidates does not exceed $T_{l 1}$, or the prefetch degree reaches the default limitation (the default is eight in our configuration, and we use the same degree adjusting technique as FDP[32]), the RLM prefetching process stops.

\subsection{Optimization}

Fast Constant Stride Prefetching: We employ a constant stride prefetcher based on the structure of HT for prefetching with invariant deltas to avoid fierce access competition of PT. If the current delta sequence consists of three identical deltas, then the delta is prefetched at a prefetch degree of three. Beyond avoiding the recursive searches of PT, it also helps in reducing the access latency for prefetching simple patterns.

\section{EVALUATION}

\subsection{Methodology}

We evaluate Matryoshka with the open-source simulator ChampSim[1]. It is the official simulator for the Data Prefetching Championship (DPC) [4, 5] used by many prefetchers[6, 7, 17, 20, 22-24, 29]. We report the IPC improvement of prefetchers normalized to the nonprefetching baseline system. Table 2 shows the simulation configuration. Please note that for single-core and 4-core systems, the settings of LLC and DRAM are different.

6.1.1 Prefetchers. We compare Matryoshka with the spatial prefetchers VLDP, SPP+PPF, Pangloss, and IPCP. VLDP implements 


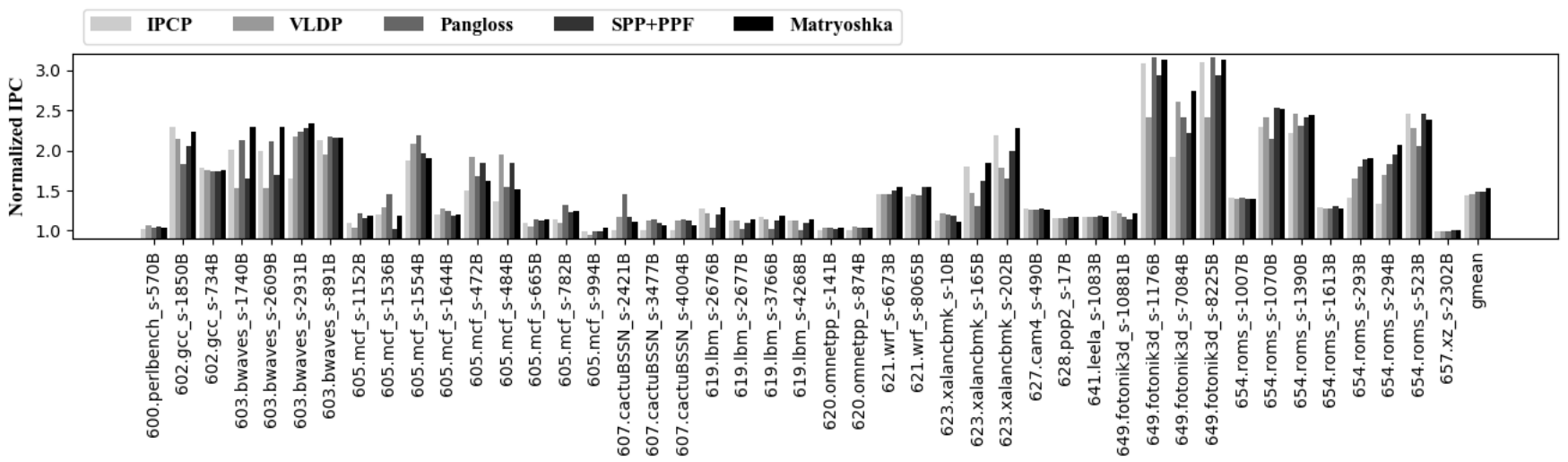

Figure 8: Detailed single-core performance comparison of L1 prefetchers.

Table 3: Prefetcher Overhead

\begin{tabular}{|c|c|c|c|c|}
\hline VLDP & SPP+PPF & Pangloss & IPCP & Matryoshka \\
\hline $48.34 \mathrm{~KB}$ & $48.39 \mathrm{~KB}$ & $45.25 \mathrm{~KB}$ & $740 \mathrm{~B}$ & $1.79 \mathrm{~KB}$ \\
\hline
\end{tabular}

the multiple matching mechanism in a simple approach and underperformed SPP according to SPP's experiments[17]. To catch up with the performance of recent prefetchers, we expand VLDP's storage capacity to $48 \mathrm{~KB}$ and apply the same optimization described in Section 5.4 to it. SPP+PPF and Pangloss are powerful but heavy prefetchers applying the conventional single matching mechanism. The Instruction Pointer Classifier based spatial Prefetching (IPCP) [22, 23], the winner of the DPC-3[5], is a low-overhead, composite prefetcher combining with three different prefetchers. Because data prefetchers improve the performance by interacting with the core and cache system, it is difficult to separately evaluate the strengths of a prefetcher. To avoid an orange to apple comparison between prefetchers, we mainly compare these prefetchers at the L1 only. Table 3 shows the overhead for all these prefetchers.

6.1.2 Workloads. We use CloudSuite[2] and 45 memory-intensive traces of SPEC 2017[3] to evaluate the performance in single-core and multi-core (4-core) systems. For single-core performance, we evaluate prefetchers using memory-intensive traces of SPEC 2017. For homogeneous multi-programmed workloads, we use traces of SPEC 2017 to generate 45 workloads with the identical benchmark for each core. For heterogeneous multi-programmed workloads, we generate 100 random mixes using traces from SPEC 2017. We also use CloudSuite to evaluate the multi-core performance. We use the first $50 \mathrm{M}$ instructions of a trace to warm up micro-architectural structures and the next $200 \mathrm{M}$ instructions to examine the performance of each core.

\subsection{Single-core Performance}

6.2.1 Performance of $L 1$ prefetchers. Fig. 8 shows the performance of prefetchers including Matryoshka, SPP+PPF, Pangloss, VLDP, and IPCP at L1. Matryoshka yields the best geometric mean speedup of $53.1 \%$ over the non-prefetching system, an improvement of $6.5 \%$ over IPCP. Compared with the conventional RLM-based prefetchers, Matryoshka outperforms SPP+PPF by $2.9 \%$ with $26 \times$ less storage and Pangloss by $3.5 \%$ with $24 \times$ less storage. Compared with the multi-matching, RLM-based prefetcher VLDP, Matryoshka surpasses it by $5.0 \%$ with $26 \times$ less storage.

Since the storage requirements of these prefetchers are different, we evaluate their performance densities[19] compared with the non-prefetching baseline to better understand the efficiency of Matryoshka. For convenience, we only consider the storage costs of caches (2640 KB for the baseline). As a result, Matryoshka improves the performance density of the baseline by $53.0 \%$, which is still $6.4 \%$ higher than IPCP. The gaps of performance densities are larger when comparing Matryoshka with heavy prefetchers (VLDP, Pangloss, and SPP+PPF), which are 6.9\%, 5.2\%, and $4.7 \%$ respectively.

According to Fig. 8, Matryoshka outperforms the other four prefetchers on 17 traces and improves the performance by several times on many traces. Besides, Matryoshka only performs the worst on one of the 45 traces. Although the enhanced VLDP implements the multi-matching mechanism in the different solution, it performs unsatisfactorily on traces of bwaves, xalancbmk, fotonik3d and roms, where Matryoshka yields $14.0 \%$ more performance. IPCP handles many kinds of situations pretty well with the composition of three different prefetchers-a stream prefetcher, a constant stride prefetcher, and a complex pattern prefetcher. It creates huge performance improvements on many traces, like gcc, bwaves, xalancbmk and fotonik3d. Matryoshka is a lightweight but powerful complex pattern prefetcher, which performs better than IPCP on these workloads by $8.1 \%$ with little more overhead. Additionally, compared with Pangloss and SPP+PPF, Matryoshka still has the upper hand in performance with way less overhead. On the 17 traces that Matryoshka performs best, the performance gaps are $12.3 \%$ and $9.0 \%$ in contrast to Pangloss and SPP+PPF.

6.2.2 Coverage, overpredictions and timeliness at $L 1$. We define covered misses as the misses reduced by a prefetcher and overpredictions as incorrect prefetches. The above metrics are normalized to the number of misses in the non-prefetching baseline system. The top sub-figure of Fig. 9 shows the coverage of five L1 prefetchers. Compared with the other prefetchers, Matryoshka achieves the highest average coverage (57.4\%) at L1, which exceeds the secondbest IPCP by $6.0 \%$. The coverage of Matryoshka is low on few workloads, but it surpasses $80 \%$ on many workloads such as gcc, bwaves, wrf, fotonik $3 d$, and roms. The coverage of VLDP is $53.6 \%$, 


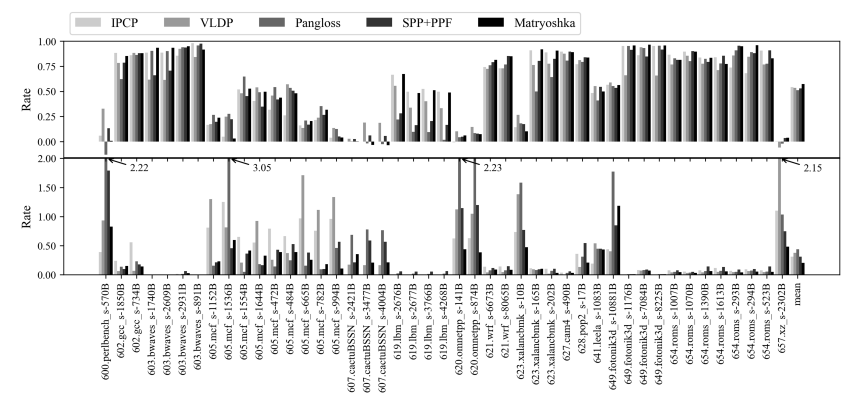

Figure 9: L1 coverage (top) and overprediction rate (bottom) comparisons of prefetchers.

which is nearly 7\% lower than that of Matryoshka. Although VLDP attempts to store key metadata using three fully-associative tables, it forbids multiple targets mapping to a unique tag, leading to a massive loss of prefetch chances.

We also measure the L1 overprediction rates for rational comparisons. The bottom sub-figure of Fig. 9 shows the results. The average overprediction rates for IPCP, VLDP, Pangloss, SPP+PPF, and Matryoshka are 30.9\%, 37.8\%, 43.7\%, 31.2\%, and 20.6\%. We find that Pangloss generates a huge amount of overpredictions on many workloads like perlbench and omnetpp. This is mainly because Pangloss tries to prefetch for every load request without tag matching. Therefore, it is easy to meet its prefetch condition, which results in the degradation of prefetch accuracy. By contrast, Matryoshka does not trigger prefetching unless matching 2-delta (20 bits) sequences, and the prefetching threshold is high, reducing many useless prefetches. Therefore, the overprediction rate is way lower than the other prefetchers despite that Matryoshka also tries to prefetch for every load access.

Timeliness is a subordinate indicator affecting performance for L1 prefetchers compared with coverage and overpredictions. The late prefetches are potentially useful prefetches that are issued just after the demands in the MSHRs. We define the prefetch-in-time rate as: useful pref/(late pref + useful pref $)$. Although all five prefetchers lack explicit prefetch timing control logic, they obtain high prefetch-in-time rates over $80 \%$, and Matryoshka achieves $87 \%$ in time prefetches.

6.2.3 Overall memory traffic. Memory traffic and overprediction rates are closely related, where useless prefetches can generate lots of additional memory accesses. Matryoshka produces $14.1 \%$ more memory traffic over the non-prefetching baseline, which is the lowest compared with the other prefetchers. Pangloss and VLDP cause $28.3 \%$ and $31.2 \%$ additional memory traffic in response to their high overprediction rates. IPCP and SPP+PPF perform better than the first two, resulting in $22.0 \%$ and $23.8 \%$ of additional memory traffic.

\subsection{Multi-core Performance}

We evaluate the multi-core performance of the five prefetchers with three types of workloads in a 4-core system. Fig. 10 shows the multi-core performance of these prefetchers. Matryoshka yields the best geometric average performance among all these multiprogrammed benchmarks and provides a $32.2 \%$ speedup over the

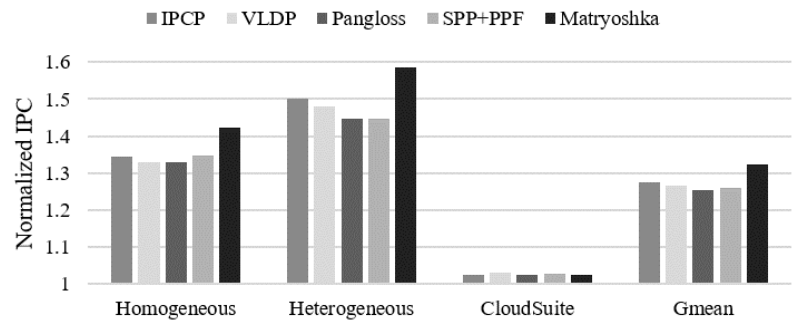

Figure 10: Summary of multi-core performance.

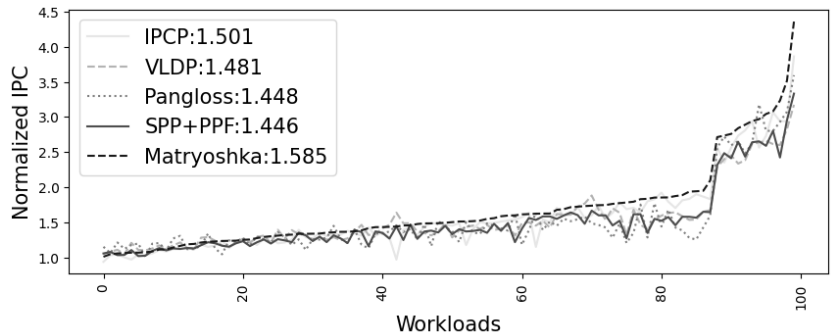

Figure 11: Detailed performance on heterogeneous workloads.

non-prefetching baseline, which is equivalent to $3.8 \%$ over IPCP and $4.4 \%$ over the enhanced VLDP.

6.3.1 Homogeneous workloads. For 4-core homogeneous workloads, Matryoshka performs better than the baseline by $42.3 \%$ and the next best prefetcher SPP+PPF by $5.7 \%$.

6.3.2 Heterogeneous workloads. Fig. 11 illustrates the detail of multi-core performance sorted in increasing order of the speedup of Matryoshka in each heterogeneous workload. Specifically, Matryoshka improves the baseline by $58.5 \%$, while outperforms SPP+PPF, Pangloss, VLDP, and IPCP by $9.6 \%, 9.4 \%, 7.0 \%$, and $5.6 \%$ respectively. In most of the heterogeneous workloads, Matryoshka achieves the best performance compared with any other prefetcher. Because the data in LLC belonging to different workloads is usually exclusive, the cache resources can be scarce. It is significant to limit the cache pollution for performance improvement in this evaluation. Due to the low overprediction rate of Matryoshka, the system utilize prefetched data efficiently, creating competitive performance.

6.3.3 CloudSuite. In general, the applications in CloudSuite are prefetch agnostic. Fig. 10 shows that VLDP obtains the best performance that improves by $3 \%$ compared with the non-prefetching baseline. The next best prefetcher is SPP+PPF that improves by $2.6 \%$ over the baseline, which is slightly over Matryoshka. On traces of the classification workload, no prefetcher performs better than the baseline.

\subsection{Comparison with VLDP}

To the best of our knowledge, VLDP is the first proposed prefetcher leveraging multi-matching of delta sequences to improve performance. The motivation of VLDP using multi-matching is to make predictions based on the longest matched delta sequence. Although Matryoshka costs much lower storage in our configuration, it outperforms VLDP in coverage and accuracy for two reasons. 


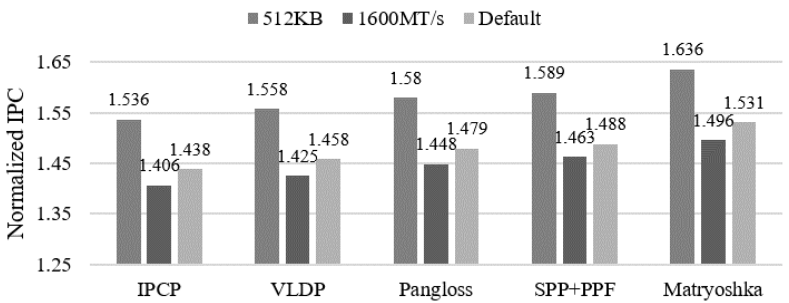

Figure 12: Performance changes under different memory bandwidths and LLC sizes.

First, Matryoshka maintains all coalesced sequences using only one table. Beyond space efficiency, it also leads to simpler updating and querying logic. Conversely, VLDP uses multiple isolated prediction tables to maintain delta sequences. This method costs more storage space for storing redundant information, and more importantly, it introduces inefficient updating and multi-set indexing logic because of cascade tables. To avoid updating multiple tables simultaneously, VLDP only updates the prediction table generating predictions last time. Because it cannot ensure that the last prediction was correct, this updating policy leads to a bias of recording, which results in the inaccurate history of delta sequences with variable lengths.

Second, because VLDP considers that longer matches of delta sequences are better than shorter ones, it does not have a precise voting mechanism for selecting prefetch candidates. But it could not be a better choice to select a longer matched sequence rarely repeated versus a shorter matched one with a higher confidence. In this situation, we think the latter is more feasible for prefetching. Matryoshka achieves higher accuracy than VLDP by addressing the above problem with the adaptive voting strategy. On the one hand, Matryoshka employs large counters to record confidences of coalesced sequences to support accurate voting results. By contrast, VLDP uses 2-bit saturated counters just for the replacement of records. On the other hand, to make use of the voting strategy, Matryoshka faithfully records history. That is, both sequences with the same prefix but different targets and sequences with the same target but different prefixes are stored to participate in voting. According to our analysis, there is an average of 3.09 short and long matches participating in the voting process each time. This result implies that there are similarities between frequently appearing sequences. Although it is suitable for VLDP to select the longest match through sequences with unique tags in each table, it leads to a loss of matching chances for accurate predictions and a decrement of coverage.

\subsection{Sensitivity Study}

6.5.1 Memory bandwidth and LLC size. Compared with the other four prefetchers, Matryoshka still obtains better performance in different configurations of the cache system. Fig. 12 shows the performance of five prefetchers with either the lower memory bandwidth or smaller LLC. Because prefetchers always need to generate more memory accesses than the baseline, so the performance is heavily impacted by the limit of bandwidths. Therefore, the normalized IPCs under $1600 \mathrm{MT} / \mathrm{s}$ bandwidth are generally lower than the situations of $3200 \mathrm{MT} / \mathrm{s}$. Despite this, Matryoshka is still the best among these prefetchers under the low bandwidth. On the other hand, results are the opposite when using a smaller LLC. Because the overpredictions of these prefetchers do not pollute caches seriously, the smaller LLC will not affect the overall performance. Fig. 12 shows that all the prefetchers obtain a relatively higher improvement with a smaller LLC. For the setting of 512KB LLC, Matryoshka obtains a $6.9 \%$ relative improvement over the setting of 2MB LLC.

6.5.2 Coalesced delta sequence length and delta width. By changing the coalesced delta sequence length and delta width of Matryoshka, we find that it is more efficient to enlarge the width of deltas. The 1delta prefix matching is disabled in all these configurations because of its negative effects on prefetch accuracy. And scoring weights are uniform for each matching length. According to the results, Matryoshka reaches the peak performance using 4-delta sequences, supporting parallel matching of 2-delta and 3-delta prefixes. Its performance is $1.2 \%$ higher than using 5 -delta sequences. The sequences that are longer than four deltas can cause more evictions during the update process. Therefore, further increasing the length is not worthy. On the other hand, increasing the width of deltas can monotonically improve performance. Although prefetching a cache block only needs seven bits for in $4 \mathrm{~KB}$ pages, the additional bits can describe patterns in the finer grain. So, much more details can be captured. For instance, 10-bit deltas can maintain patterns in spatial correlations of 8-byte blocks. This finer-grained description yields a speedup of $1.0 \%$ by using 7 -bit deltas. We also enlarge the delta width of VLDP to measure its sensitivity. When enlarging its delta width to 10 bits from 7 bits which leads to a storage consumption of $63 \mathrm{~KB}$ (even larger than the L1D), Matryoshka still outperforms it by about $3 \%$.

6.5.3 Multi-hierarchy prefetcher. IPCP leverages the communication interface between L1 and L2 to build an efficient L2 constant stride prefetcher (costs 155B). We equip Matryoshka with a similar helper prefetcher at L2 (costs 64B) to explore its multi-hierarchy scalability. Experimental results show that Matryoshka improves the performance of the L1 edition by $4.6 \%$, which performs better than IPCP by $1.5 \%$ in the multi-hierarchy environment.

6.5.4 Storage. Benefiting from the dynamic indexing strategy, Matryoshka preserves the key information costing small storage space without intensive hash conflicts. Enlarging the storage of Matryoshka can reduce evictions of coalesced sequences. But we find it contributes little to performance improvement. Through the experiment, the performance improves by about $1.5 \%$ when enlarging the storage to about $50 \times(2 \mathrm{~K}$ entries for $\mathrm{HT}$ and $256 \times 64$ entries for PT).

\section{CONCLUSION}

Often, there can be considerable memory access patterns in various workloads to be captured for prefetching. Due to the variety of complex patterns, it is inadequate to recognize them accurately using the conventional matching strategy. We first present the approach of coalescing metadata to improve the performance and storage efficiency of a prefetcher and propose Matryoshka that recognizes the complex patterns through coalesced delta sequences. Through comparative experiments, Matryoshka obtains a speedup over the state-of-the-art prefetcher IPCP by $6.5 \%$ at the cost of 
$1.79 \mathrm{~KB}$ and outperforms the high-performance heavy prefetchers with $20 \times$ lower storage space.

Looking to the future, we tend to exploit the spatial correlations between physical pages. After some early explorations, we have found there are massive repetitions of deltas between pages, which indicates a possibility of prefetching addresses that cross pages. We plan to design a novel low-overhead prefetcher, leveraging deltas inner pages and inter pages to obtain a further improvement of performance.

\section{ACKNOWLEDGMENTS}

We thank the anonymous reviewers for their feedback. We also thank Professor Radu Teodorescu for his selfless help in the revision of the paper.

\section{REFERENCES}

[1] [n.d.]. ChampSim simulator. https://github.com/ChampSim/ChampSim

[2] [n.d.]. Cloudsuite traces. https://www.dropbox.com/sh/pgmnzfr3hurlutq/ AACciuebRwSAOzhJkmj5SEXBa/CRC2_trace?dl=0\&subfolder_nav_tracking $=1$

[3] [n.d.]. Spec cpu 2017 traces (spec speed: 6xx numbered). http://hpca23.cse.tamu. edu/champsim-traces/speccpu/

[4] 2015. The 2nd Data Prefetching Championship. http://comparch-conf.gatech $\mathrm{edu} / \mathrm{dpc} 2 /$

[5] 2019. The 3rd Data Prefetching Championship. https://dpc3.compas.cs. stonybrook.edu/

[6] M. Bakhshalipour, M. Shakerinava, P. Lotfi-Kamran, and H. Sarbazi-Azad. 2019 Bingo Spatial Data Prefetcher. In 2019 IEEE International Symposium on High Performance Computer Architecture (HPCA). 399-411.

[7] E. Bhatia, G. Chacon, S. Pugsley, E. Teran, P. V. Gratz, and D. A. Jiménez. 2019 Perceptron-Based Prefetch Filtering. In 2019 ACM/IEEE 46th Annual International Symposium on Computer Architecture (ISCA). 1-13.

[8] Jason F. Cantin, Mikko H. Lipasti, and James E. Smith. 2006. Stealth prefetching. In Proceedings of the 12th International Conference on Architectural Support for Programming Languages and Operating Systems, ASPLOS 2006, San fose, CA, USA, October 21-25, 2006, John Paul Shen and Margaret Martonosi (Eds.). ACM, 274-282. https://doi.org/10.1145/1168857.1168892

[9] Chi F. Chen, Se-Hyun Yang, Babak Falsafi, and Andreas Moshovos. 2004. Accurate and Complexity-Effective Spatial Pattern Prediction. In 10th International Conference on High-Performance Computer Architecture (HPCA-10 2004), 14-18 February 2004, Madrid, Spain. IEEE Computer Society, 276-287. https: //doi.org/10.1109/HPCA.2004.10010

[10] Tien-Fu Chen and Jean-Loup Baer. 1995. Effective Hardware Based Data Prefetching for High-Performance Processors. IEEE Trans. Computers 44, 5 (1995), 609-623. https://doi.org/10.1109/12.381947

[11] Keith I. Farkas, Paul Chow, Norman P. Jouppi, and Zvonko G. Vranesic. 1997. Memory-System Design Considerations for Dynamically-Scheduled Processors. In Proceedings of the 24th International Symposium on Computer Architecture Denver, Colorado, USA, June 2-4, 1997, Andrew R. Pleszkun and Trevor N. Mudge (Eds.). ACM, 133-143. https://doi.org/10.1145/264107.264156

[12] Ibrahim Hur and Calvin Lin. 2006. Memory Prefetching Using Adaptive Stream Detection. In 39th Annual IEEE/ACM International Symposium on Microarchitecture (MICRO-39 2006), 9-13 December 2006, Orlando, Florida, USA. IEEE Computer Society, 397-408. https://doi.org/10.1109/MICRO.2006.32

[13] Yasuo Ishii, Mary Inaba, and Kei Hiraki. 2009. Access map pattern matching for data cache prefetch. In Proceedings of the 23rd international conference on Supercomputing, 2009, Yorktown Heights, NY, USA, June 8-12, 2009, Michael Gschwind, Alexandru Nicolau, Valentina Salapura, and José E. Moreira (Eds.). ACM, 499-500. https://doi.org/10.1145/1542275.1542349

[14] Yasuo Ishii, Mary Inaba, and Kei Hiraki. 2011. Access Map Pattern Matching for High Performance Data Cache Prefetch. F. Instr. Level Parallelism 13 (2011). http://www.jilp.org/vol13/v13paper3.pdf

[15] Teresa L. Johnson, Matthew C. Merten, and Wen-mei W. Hwu. 1997. Run-Time Spatial Locality Detection and Optimization. In Proceedings of the Thirtieth Annual IEEE/ACM International Symposium on Microarchitecture, MICRO 30, Research Triangle Park, North Carolina, USA, December 1-3, 1997, Mark Smotherman and Tom Conte (Eds.). ACM/IEEE Computer Society, 57-64. https://doi.org/10.1109/ MICRO.1997.645797

[16] Norman P. Jouppi. 1990. Improving Direct-Mapped Cache Performance by the Addition of a Small Fully-Associative Cache and Prefetch Buffers. In Proceedings of the 17th Annual International Symposium on Computer Architecture, Seattle, WA, USA, fune 1990, Jean-Loup Baer, Larry Snyder, and James R. Goodman (Eds.)
ACM, 364-373. https://doi.org/10.1145/325164.325162

[17] J. Kim, S. H. Pugsley, P. V. Gratz, A. L. N. Reddy, C. Wilkerson, and Z. Chishti. 2016. Path confidence based lookahead prefetching. In 2016 49th Annual IEEE/ACM International Symposium on Microarchitecture (MICRO). 1-12.

[18] Sanjeev Kumar and Christopher B. Wilkerson. 1998. Exploiting Spatial Locality in Data Caches Using Spatial Footprints. In Proceedings of the 25th Annual International Symposium on Computer Architecture, ISCA 1998, Barcelona, Spain, Fune 27 - Fuly 1, 1998, Mateo Valero, Gurindar S. Sohi, and Doug DeGroot (Eds.). IEEE Computer Society, 357-368. https://doi.org/10.1109/ISCA.1998.694794

[19] Pejman Lotfi-Kamran, Boris Grot, Michael Ferdman, Stavros Volos, Yusuf Onur Koçberber, Javier Picorel, Almutaz Adileh, Djordje Jevdjic, Sachin Idgunji, Emre Özer, and Babak Falsafi. 2012. Scale-out processors. In 39th International Symposium on Computer Architecture (ISCA 2012), June 9-13, 2012, Portland, OR, USA. IEEE Computer Society, 500-511. https://doi.org/10.1109/ISCA.2012.6237043

[20] Pierre Michaud. 2016. Best-offset hardware prefetching. In 2016 IEEE International Symposium on High Performance Computer Architecture, HPCA 2016, Barcelona, Spain, March 12-16, 2016. IEEE Computer Society, 469-480. https://doi.org/10. 1109/HPCA.2016.7446087

[21] Celal Öztürk, Ibrahim Burak Karsli, and Resit Sendag. 2014. An analysis of address and branch patterns with PatternFinder. In 2014 IEEE International Symposium on Workload Characterization, IISWC 2014, Raleigh, NC, USA, October 26-28, 2014. IEEE Computer Society, 232-242. https://doi.org/10.1109/IISWC.2014.6983062

[22] Samuel Pakalapati and Biswabandan Panda. 2019. Bouquet of Instruction Pointers: Instruction Pointer Classifier based Hardware Prefetching. In Proceedings of the 3rd Data Prefetching Championship. https://dpc3.compas.cs.stonybrook.edu/

[23] Samuel Pakalapati and Biswabandan Panda. 2020. Bouquet of Instruction Pointers: Instruction Pointer Classifier-based Spatial Hardware Prefetching. In 47th ACM/IEEE Annual International Symposium on Computer Architecture, ISCA 2020, Valencia, Spain, May 30 - June 3, 2020. IEEE, 118-131. https://doi.org/10.1109/ ISCA45697.2020.00021

[24] Philippos Papaphilippou, Paul H. J. Kelly, and Wayne Luk. 2019. Pangloss: a novel Markov chain prefetcher. CoRR abs/1906.00877 (2019). arXiv:1906.00877 http://arxiv.org/abs/1906.00877

[25] Seth H. Pugsley, Zeshan Chishti, Chris Wilkerson, Peng-fei Chuang, Robert L. Scott, Aamer Jaleel, Shih-Lien Lu, Kingsum Chow, and Rajeev Balasubramonian. 2014. Sandbox Prefetching: Safe run-time evaluation of aggressive prefetchers. In 20th IEEE International Symposium on High Performance Computer Architecture, HPCA 2014, Orlando, FL, USA, February 15-19, 2014. IEEE Computer Society, 626-637. https://doi.org/10.1109/HPCA.2014.6835971

[26] Andre Seznec. 2014. Tage-sc-1 branch predictors. In Proceedings of the 4th Championship on Branch Prediction. http://www.jilp.org/cbp2014/

[27] Andre Seznec. 2016. Tage-sc-l branch predictors again. In Proceedings of the 5th Championship on Branch Prediction. http://www.jilp.org/cbp2016/

[28] André Seznec and Pierre Michaud. 2006. A case for (partially) TAgged GEometric history length branch prediction. F. Instr. Level Parallelism 8 (2006). http: //www.jilp.org/vol8/v8paper1.pdf

[29] Manjunath Shevgoor, Sahil Koladiya, Rajeev Balasubramonian, Chris Wilkerson, Seth H. Pugsley, and Zeshan Chishti. 2015. Efficiently prefetching complex address patterns. In Proceedings of the 48th International Symposium on Microarchitecture, MICRO 2015, Waikiki, HI, USA, December 5-9, 2015, Milos Prvulovic (Ed.). ACM, 141-152. https://doi.org/10.1145/2830772.2830793

[30] Alan Jay Smith. 1978. Sequential Program Prefetching in Memory Hierarchies. Computer 11, 12 (1978), 7-21. https://doi.org/10.1109/C-M.1978.218016

[31] Stephen Somogyi, Thomas F. Wenisch, Anastassia Ailamaki, Babak Falsafi, and Andreas Moshovos. 2006. Spatial Memory Streaming. In Proceedings of the 33rd Annual International Symposium on Computer Architecture (ISCA '06). IEEE Computer Society, USA, 252-263. https://doi.org/10.1109/ISCA.2006.38

[32] Santhosh Srinath, Onur Mutlu, Hyesoon Kim, and Yale N. Patt. 2007. Feedback Directed Prefetching: Improving the Performance and Bandwidth-Efficiency of Hardware Prefetchers. In 13st International Conference on High-Performance Computer Architecture (HPCA-13 2007), 10-14 February 2007, Phoenix, Arizona, USA. IEEE Computer Society, 63-74. https://doi.org/10.1109/HPCA.2007.346185

[33] Hao Wu, Krishnendra Nathella, Joseph Pusdesris, Dam Sunwoo, Akanksha Jain, and Calvin Lin. 2019. Temporal Prefetching Without the Off-Chip Metadata. In Proceedings of the 52nd Annual IEEE/ACM International Symposium on Microarchitecture, MICRO 2019, Columbus, OH, USA, October 12-16, 2019. ACM, 996-1008. https://doi.org/10.1145/3352460.3358300

[34] William A. Wulf and Sally A. McKee. 1995. Hitting the memory wall: implications of the obvious. SIGARCH Comput. Archit. News 23, 1 (1995), 20-24. https: //doi.org/10.1145/216585.216588 